\title{
A Brazilian-Portuguese version of the Kinesthetic and Visual Motor Imagery Questionnaire
}

\author{
Versão brasileira do Questionário de Imagética Motora Cinestésica e Visual \\ Alan Demanboro', Annette Sterr ${ }^{1,2}$, Sarah Monteiro dos Anjos ${ }^{1,3}$, Adriana Bastos Conforto ${ }^{1,4}$
}

\begin{abstract}
Motor imagery has emerged as a potential rehabilitation tool in stroke. The goals of this study were: 1) to develop a translated and culturally-adapted Brazilian-Portugese version of the Kinesthetic and Visual Motor Imagery Questionnaire (KVIQ20-P); 2) to evaluate the psychometric characteristics of the scale in a group of patients with stroke and in an age-matched control group; 3 ) to compare the KVIQ20 performance between the two groups. Methods: Test-retest, inter-rater reliabilities, and internal consistencies were evaluated in 40 patients with stroke and 31 healthy participants. Results: In the stroke group, ICC confidence intervals showed excellent test-retest and inter-rater reliabilities. Cronbach's alpha also indicated excellent internal consistency. Results for controls were comparable to those obtained in persons with stroke. Conclusions: The excellent psychometric properties of the KVIQ20-P should be considered during the design of studies of motor imagery interventions for stroke rehabilitation.
\end{abstract}

Keywords: rehabilitation; paresis; stroke; reproducibility of results.

\section{RESUMO}

A Imagética Mental é uma ferramenta em potencial para a reabilitação de indivíduos com doenças cerebrovasculares. Os objetivos desse estudo foram: 1) Desenvolver uma versão traduzida e adaptada culturalmente do Questionário de Imagética Motora Cinestésica e Visual (KVIQ20-P); 2) Avaliar as características psicométricas da escala nesse grupo e em controles; 3) Comparar o desempenho na escala entre os dois grupos. Métodos: Confiabilidades teste-reteste, interexaminador, assim como consistências internas da KVIQ20-P foram avaliadas em 40 indivíduos com AVC e em 31 controles. Resultados: No grupo de pacientes, os intervalos de confiança mostraram excelentes confiabilidades teste-reteste e interexaminador. 0 alfa de Cronbach indicou uma excelente consistência interna. Os resultados no grupo controle foram comparáveis aos obtidos nos pacientes. Conclusões: As excelentes propriedades psicométricas da KVIQ20-P devem ser consideradas durante o desenho de estudos de Imagética Mental para a reabilitação de indivíduos com doenças cerebrovasculares.

Palavras-chave: reabilitação; paresia; acidente vascular cerebral; reprodutibilidade dos testes.

Motor imagery consists of mentally evoking a motor act without actually doing it. The neural representation of motor acts, as well as their visual and kinesthetic properties, are therefore activated through the mental process of imagination $^{1,2}$. Perception, action, and imagery share common neural pathways involving mainly the frontal and parietal areas of the brain ${ }^{3,4,5,6,7}$. Considering the latest developments in the field, there is no consensus on the level of imagery proficiency required to perform motor imagery interventions effectively.
There are large differences in performance among individuals, and various instruments have been developed to measure motor imagery ability. Among these instruments, the Kinesthetic and Visual Motor Imagery Questionnaire (KVIQ) is widely used ${ }^{1,8,9}$. The KVIQ consists of 20 items $\left(\mathrm{KVIQ}_{20}\right)$ clustered into two 10-item subscales (visual, $\mathrm{KVIQ}_{10 \mathrm{~V}}$ and kinesthetic, $\mathrm{KVIQ}_{10 \mathrm{~K}}$ ). The $\mathrm{KVIQ}_{20}$ scores range from 20 to 100 , while the $\mathrm{KVIQ}_{10 \mathrm{~V}}$ and $\mathrm{KVIQ}_{10 \mathrm{~K}}$ scores each range from 10 to 50 , with greater scores indicating greater aptitude in motor imagery.

\footnotetext{
${ }^{1}$ Universidade de São Paulo, Hospital das Clinicas, São Paulo SP, Brasil;

${ }^{2}$ University of Surrey, School of Psychology, UK;

${ }^{3}$ University of Alabama at Birmingham, School of Health Professions, Departments of Physical Therapy and Occupational Therapy, Birmingham, AL;
}

${ }^{4}$ Hospital Israelita Albert Einstein, São Paulo SP, Brasil.

Support: Annette Sterr received funding from the Science Without Borders program from Coordenação de Aperfeiçoamento de Pessoal de Nivel Superior - CAPES (Grant number:A068/2013). CAPES had no role in the design of the study or the collection, analysis, and interpretation of data, or in writing the manuscript.

Correspondence: Adriana B. Conforto; Divisão de Neurologia Clínica, Hospital das Clínicas da Universidade de São Paulo; Av. Dr. Enéas de Carvalho Aguiar, 255 / 5084; 05410-030 São Paulo SP, Brasil; E-mail: adriana.conforto@gmail.com

Conflict of interest: There is no conflict of interest:

Received 11 August 2017; Received in final form 09 October 2017; Accepted 23 October 2017. 
This scale was specifically created for people with physical disabilities $^{1}$ and has been reliable when applied to individuals with stroke, in different parts of the world ${ }^{1,8}$.

Motor imagery is potentially a very attractive rehabilitation concept for countries facing substantial challenges in the provision of stroke rehabilitation. One of the critical aspects in this endeavor is the availability of adequate measures of the extent of motor imagery in persons with stroke. The strong evidence base using the KVIQ scale in Englishspeaking countries makes it an excellent candidate for this purpose. Therefore, the present study comprised: 1) the development of a translated and culturally adapted BrazilianPortugese version of the KVIQ (KVIQ-P); 2) evaluation of the psychometric characteristics of the scale in the stroke group and in an age-matched control group; 3) comparison of the KVIQ-P performance in persons with stroke and in controls.

\section{METHOD}

\section{Subjects}

For the stroke group, participants were recruited through databases of the Stroke Group at Hospital das Clínicas/São Paulo University and a primary care clinic in the São Paulo metropolitan area between December, 2010 and April, 2014. The candidates were contacted and invited to complete a telephone screening. Eligible participants were then invited for testing in the neurostimulation laboratory at Hospital das Clínicas. Age- and gender-matched controls were recruited among acquaintances of the participants or researchers.

Inclusion criteria for the stroke group were: a minimum age of 21 years, single unilateral ischemic stroke in one cerebral hemisphere leading to hemiparesis, more than six months before and confirmed by computed tomography or magnetic resonance imaging; ability to understand the protocol and provide written informed consent. The exclusion criteria were: Mini-Mental State Examination (MMSE) scores lower than the cut-offs for literate (24/30) or illiterate adults (20/30; it is estimated that $8.3 \%$ of Brazilians older than 15 years are illiterate $)^{10,11}$; use of drugs affecting cognition or balance such as benzodiazepines, neuroleptics and antiepileptic drugs ${ }^{12}$; history of other neurological conditions; uncontrolled medical disease; blindness ${ }^{13}$ and severe congenital or acquired visual deficits; history of depression and alcohol abuse, evaluated with DSM-V criteria ${ }^{14}$, and other psychiatric conditions such as psychosis or schizophrenia reported by participants and/or their caregivers. Because the study provided groundwork for a project on motor imagery in lower limb recovery, we also excluded participants with a Berg Balance Scale $>54$ points, as lower scores are associated with an increased risk of falls (maximum score, 56 points) ${ }^{15}$ and the goal of the rehabilitation project was to improve balance and decrease this risk. Figure 1 shows the flow of persons with stroke throughout the study.

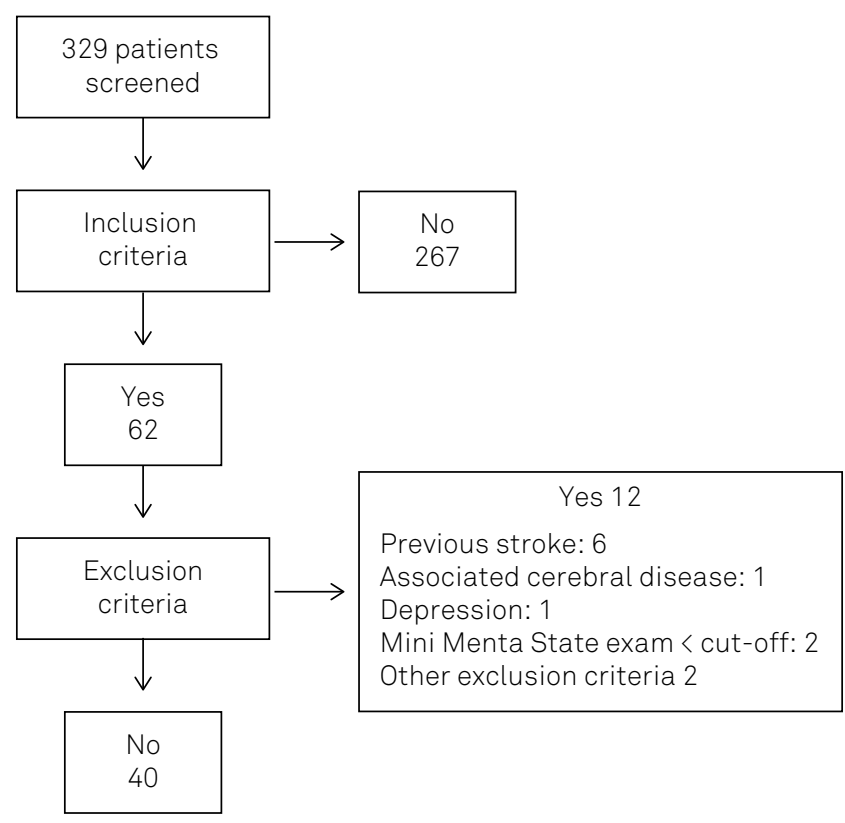

Figure. Flow of participants through the study.

To test the psychometric properties of the KVIQ-P in controls, as well as to compare performances between persons with stroke and controls, a healthy control group with comparable demographic characteristics $(n=31)$ was also recruited. Inclusion criteria were: minimum age of 21 years; ability to understand the protocol and provide written informed consent. Exclusion criteria were: history of stroke and the same criteria applied to the stroke group.

The protocol was approved by the Ethics Committee of the Hospital das Clinicas/São Paulo University (protocol number 0471/09) and all participants provided written informed consent.

\section{Translation and cultural adaptation}

We followed the guiding principles for cultural adaptation of assessment instruments ${ }^{16-19}$. Two independent Brazilian specialists in the English language translated the original $\mathrm{KVIQ}_{20}$ version into Brazilian Portuguese. Two independent Brazilian-Portuguese health professionals with English proficiency (a psychologist and a physical therapist) did the back-translation. The versions were compared to check the linguistic equivalence and consistency and a single version was defined by consensus between native speakers of Portuguese.

In the next step, this version of the $\mathrm{KVIQ}_{20}$ was piloted in five participants with stroke. Based on the feedback obtained from these participants in a post-testing interview regarding clarity and understandability of the instrument, the questions were refined and finalized to the Brazilian-Portuguese version of the KVIQ administered for the study (KVIQ-P; supplementary file).

Movements imagined in the KVIQ include actions of all body segments. There are specific tests for imagined 
movements of the upper limbs, tests for imagined movements of the lower limbs, as well as the neck and trunk. All movements are executed, and then imagined while the subject is seated.

\section{Determination of psychometric properties of the $K V I Q-P_{20}, K V I Q-P_{10 V}$ and $K V I Q-P_{10 K}$}

Three parameters, internal consistency, intra-rater and inter-rater reliability were determined for the stroke and the control groups respectively. For intra-rater reliability, the participants were retested within one to two weeks of the first test. All evaluations were videotaped and rated by Researcher 1. For inter-rater reliability, Researcher 2 rated the videotaped performance of the first test, and the scores of the two raters were compared. Internal consistencies of the KVIQ-P ${ }_{20}$, KVIQ-P ${ }_{10 \mathrm{~V}}$ and KVIQ-P ${ }_{10 \mathrm{~K}}$ were calculated.

\section{Comparison between the KVIQ performance in persons with stroke and controls}

The KVIQ-P ${ }_{20}$, KVIQ-P ${ }_{10 V}$ and KVIQ-P ${ }_{10 K}$ scores of the first evaluations of the stroke group and control group were compared. Considering that people with stroke often had deficits such as dysarthria, hemiparesis or aphasia, among others, blinding of the evaluations was not possible.

\section{Statistics}

Data were described by means \pm standard deviations for normally-distributed variables, and by medians and ranges if otherwise. Frequencies were calculated for categorical variables. The Kolmogorov-Smirnov test revealed that the data were not normally distributed and hence nonparametric tests were used for within-subject comparisons (performances in the KVIQ- $\mathrm{P}_{20}$, KVIQ-P ${ }_{10 V}$ and KVIQ- $\mathrm{P}_{10 \mathrm{~K}}$ : Wilcoxon test) and between-group comparisons (KVIQ- $\mathrm{P}_{20}$, KVIQ-P $\mathrm{P}_{10 \mathrm{~V}}$ and KVIQ-P $\mathrm{P}_{10 \mathrm{~K}}$ : Mann-Whitney tests).

Internal consistencies of the KVIQ- $\mathrm{P}_{20}$ scale, as well as their subscales were evaluated with Cronbach's alpha test ${ }^{20}$. The score of each item was compared with the total score by Cronbach's alpha correlation if an item was deleted. Consistencies were classified according to Bland and Altman ${ }^{20}$. A Cronbach's alpha between 0.70 and 0.80 is considered satisfactory for a reliable comparison between groups and a minimum of 0.90 is required for a scale used for clinical purposes, while values of at least 0.95 are considered desirable.

Intra-rater reliability (first application of the scale vs. second application) and inter-rater reliability (Researcher 1 direct evaluation of the first application vs. evaluation of the videotapes of the first application by Researcher 2) were analyzed with the Intraclass Correlation Coefficient test (ICC) applied in a two-way random model with total agreement and classified $\mathrm{as}^{21}$ : ICC $<0.40=$ poor reliability; ICC $\geq 0.40$ but $\leq 0.75=$ fair to good reliability; and ICC $>0.75=$ excellent reliability. The ICCs for the KVIQ- ${ }_{20}$ and its subscales (KVIQ-P ${ }_{10 \mathrm{~V}}$ and KVIQ-P ${ }_{10 K}$ ) were determined.
Inter-item correlations were calculated if an item was deleted to search for items that could cause inconsistencies because of extreme values. Values of $0.40-0.50$ inter-item correlation are required for scales tapping narrower characteristics, as is the case of the KVIQ- $\mathrm{P}_{20}$, based on fewer items ${ }^{22}$.

All data were tested with the SPSS18 statistical software.

\section{RESULTS}

\section{Characteristics of the participants}

Table 1 gives an overview of participant characteristics for both groups. There were no significant differences between demographic characteristics of persons with stroke and the controls $(p>0.05)$. A large proportion was under the age of $65: n=33(82.5 \%)$ in the stroke group, and $n=24(77.4 \%)$ in the control group. Twenty-eight (70\%) of the participants in the stroke group, and 21 (67.7\%) of the controls had eight years of education or more. The mean time from stroke $( \pm$ standard deviation) was $3.6 \pm 2.2$ years. In $62.5 \%$ of the persons with stroke, lesions were located in the right hemisphere. The mean Berg Balance Scale score was $44.7 \pm 9$ in the stroke group.

\section{Psychometric properties}

The internal consistency of the KVIQ- $\mathrm{P}_{20}$ and the consistency of each subscale are presented in Table2.Intra-rater and inter-rater reliabilities of the KVIQ- $\mathrm{P}_{20}$, as well as the KVIQ- $\mathrm{P}_{10 \mathrm{~V}} / \mathrm{KVIQ}-\mathrm{P}_{10 \mathrm{~K}}$ subscales, were excellent according to the criteria adopted in both groups. Table 3 shows inter-item correlations.

\section{Comparison between the KVIQ-P performance in persons with stroke and controls}

The KVIQ-P performance was comparable between the stroke and control groups for the KVIQ- $\mathrm{P}_{20}(66.0 \pm 16.3$ / stroke group; $66.1 \pm 21.3$ /control group), KVIQ-P ${ }_{10 \mathrm{~V}}(32.7 \pm$ 9.6/stroke group; $33.0 \pm 11.8$ /control group) and KVIQ-P ${ }_{10 \mathrm{~K}}$ (33.1 \pm 8.5 /stroke group; $33.1 \pm 10.6 /$ control group). None of these numerical differences between groups were statistically significant (KVIQ- ${ }_{20}, \mathrm{p}=0.68$; KVIQ-P ${ }_{10 \mathrm{v}} \mathrm{p}=0.80$; KVIQ-P ${ }_{10 K}-\mathrm{p}=0.61$ ).

Table 1. Characteristics of the participants. Means and standard deviations or medians and ranges are given.

\begin{tabular}{lcc}
\hline Characteristics & Stroke group & Control group \\
\hline Age (years) & $54.8 \pm 12.6$ & $55.2 \pm 12.9$ \\
\hline Education (years) & $9.3 \pm 4.9$ & $9.4 \pm 4.6$ \\
Mini-mental state examination & $27(20-30)$ & $29(25-30)$ \\
$K V I Q-P_{20}$ & $66.0 \pm 16.3$ & $66.1 \pm 21.3$ \\
$K V I Q-P_{10 \mathrm{~V}}$ & $32.7 \pm 9.6$ & $33.0 \pm 11.8$ \\
$\mathrm{KVIQ}-\mathrm{P}_{10 \mathrm{~K}}$ & $33.1 \pm 8.5$ & $33.1 \pm 10.6$ \\
\hline $\mathrm{KVIQ}-\mathrm{P}_{20}:$ kinesthetic and visual motor imagery questionnaire - full scale; \\
$\mathrm{KVIQ}-\mathrm{P}_{10 \mathrm{v}}:$ kinesthetic and visual motor imagery questionnaire - visual \\
subscale; $\mathrm{KVIQ}-\mathrm{P}_{10 \mathrm{~K}}:$ kinesthetic and visual
\end{tabular}


Table 2. Reliability of the Brazilian version of the Kinesthetic and Visual Motor Imagery Questionnaire $\left(K V I Q-P_{20}\right)$ and its subscales.

\begin{tabular}{|c|c|c|c|c|c|c|}
\hline \multirow{2}{*}{ Reliability } & \multicolumn{3}{|c|}{ Stroke Group } & \multicolumn{3}{|c|}{ Control Group } \\
\hline & $K V I Q-P_{20}$ & $K V I Q-P_{10 V}$ & $K V I Q-P_{10 K}$ & $K V I Q-P_{20}$ & $K V I Q-P_{10 V}$ & $K V I Q-P_{10 K}$ \\
\hline \multirow{3}{*}{ Intra-rater } & $I C C=0.85$ & $\mathrm{ICC}=0.87$ & $I C C=0.75$ & $I C C=0.90$ & $I C C=0.90$ & $\mathrm{ICC}=0.82$ \\
\hline & $(0.74-0.92)$ & $(0.77-0.93)$ & $(0.57-0.86)$ & $(0.81-0.95)$ & $(0.81-0.95)$ & $(0.67-0.91)$ \\
\hline & $p<0.001$ & $p<0.001$ & $p<0.001$ & $p<0.001$ & $p<0.001$ & $p<0.001$ \\
\hline \multirow{3}{*}{ Inter-rater } & $I C C=0.99$ & ICC = 0.99 & ICC -0.99 & ICC -0.99 & ICC -0.99 & ICC -0.99 \\
\hline & $(0.99-1.00)$ & $(0.99-1.00)$ & $(0.99-0.99)$ & $(0.99-0.99)$ & $(0.99-0.99)$ & $(0.99-0.99)$ \\
\hline & $p<0.001$ & $p<0.001$ & $p<0.001$ & $p<0.001$ & $p<0.001$ & $p<0.001$ \\
\hline Internal consistency & $\alpha=0.94$ & $\alpha=0.95$ & $\alpha=0.94$ & $\alpha=0.97$ & $\alpha=0.97$ & $\alpha=0.95$ \\
\hline
\end{tabular}

$\mathrm{KVIQ}-\mathrm{P}_{20}:$ kinesthetic and visual motor imagery questionnaire - full scale; KVIQ- ${ }_{10 \mathrm{v}}$ : kinesthetic and visual motor imagery questionnaire - visual subscale; $K V I Q-P_{10 K}^{20}:$ kinesthetic and visual motor imagery questionnaire - kinesthetic subscale; ICC: intraclass coefficient correlation.

Table 3. Internal consistency. Cronbach's alpha for correlation between the KVIQ- $P_{20}$ score, KVIQ- $P_{10 V}$ and $K V I Q-P_{10 K} S c 0 r e s$ if item deleted.

\begin{tabular}{|c|c|c|c|c|c|c|c|}
\hline \multicolumn{4}{|c|}{ Stroke group } & \multicolumn{4}{|c|}{ Control group } \\
\hline \multirow{2}{*}{ Item } & $\begin{array}{l}\mathrm{KVIQ}_{20} \text { Cronbach's alpha } \\
\text { if item deleted }\end{array}$ & \multirow{2}{*}{ Item } & $\begin{array}{l}\text { Cronbach's alpha if } \\
\text { item deleted }\end{array}$ & \multirow[t]{2}{*}{ Item } & \multirow{2}{*}{$\begin{array}{c}\mathrm{KVIQ}_{20} \text { Cronbach's alpha } \\
\text { if item deleted } \\
\mathrm{KVIQ} \mathrm{Q}_{20}\end{array}$} & \multirow{2}{*}{ Item } & $\begin{array}{l}\text { Cronbach's alpha if } \\
\text { item deleted }\end{array}$ \\
\hline & $\mathrm{KVIQ}_{20}$ & & $\mathrm{KVIQ}_{10 \mathrm{~V}}$ & & & & $\mathrm{KVIQ}_{10 \mathrm{~V}}$ \\
\hline V01 & 0.941 & V01 & 0.930 & V01 & 0.974 & V01 & 0.970 \\
\hline V02 & 0.938 & V02 & 0.929 & V02 & 0.975 & V02 & 0.971 \\
\hline V03 & 0.936 & V03 & 0.919 & V03 & 0.974 & V03 & 0.969 \\
\hline V04 & 0.936 & V04 & 0.916 & V04 & 0.973 & V04 & 0.969 \\
\hline V05 & 0.936 & V05 & 0.916 & V05 & 0.973 & V05 & 0.968 \\
\hline V06 & 0.936 & V06 & 0.917 & V06 & 0.975 & V06 & 0.972 \\
\hline V07 & 0.938 & V07 & 0.923 & V07 & 0.975 & V07 & 0.969 \\
\hline V08 & 0.937 & V08 & 0.925 & V08 & 0.974 & V08 & 0.968 \\
\hline V09 & 0.936 & V09 & 0.923 & V09 & 0.975 & V09 & 0.969 \\
\hline V10 & 0.934 & V10 & 0.916 & V10 & 0.974 & V10 & 0.969 \\
\hline Item & $\mathrm{KVIQ}_{20}$ & & $K V I Q_{10 K}$ & & $\mathrm{KVIQ}_{20}$ & & $K V I Q_{10 K}$ \\
\hline K01 & 0.939 & K01 & 0.904 & K01 & 0.976 & K01 & 0.953 \\
\hline K02 & 0.938 & K02 & 0.893 & K02 & 0.976 & K02 & 0.956 \\
\hline K03 & 0.936 & K03 & 0.885 & K03 & 0.974 & K03 & 0.952 \\
\hline K04 & 0.937 & K04 & 0.886 & K04 & 0.974 & K04 & 0.950 \\
\hline K05 & 0.938 & K05 & 0.891 & K05 & 0.974 & K05 & 0.954 \\
\hline K06 & 0.936 & K06 & 0.892 & K06 & 0.976 & K06 & 0.957 \\
\hline K07 & 0.938 & K07 & 0.893 & K07 & 0.974 & K07 & 0.949 \\
\hline K08 & 0.940 & K08 & 0.904 & K08 & 0.974 & K08 & 0.950 \\
\hline K09 & 0.939 & K09 & 0.898 & K09 & 0.974 & K09 & 0.949 \\
\hline K10 & 0.937 & K10 & 0.894 & K10 & 0.973 & K10 & 0.946 \\
\hline
\end{tabular}

$\mathrm{KVIQ}-\mathrm{P}_{2}$ : kinesthetic and visual motor imagery questionnaire - full scale; $\mathrm{KVIQ}-\mathrm{P}_{10 \mathrm{~V}}$ : kinesthetic and visual motor imagery questionnaire - visual subscale; $K V I Q-P_{10 K}^{20}:$ kinesthetic and visual motor imagery questionnaire - kinesthetic subscale.

\section{DISCUSSION}

The current research aimed to adapt and evaluate the psychometric properties of a Brazilian-Portuguese version of the most commonly-used motor imagery questionnaire in English-speaking countries, the KVIQ-P. Overall, the results of this study suggest that the Brazilian-Portuguese version of the KVIQ has strong psychometric characteristics. Specifically, we obtained excellent intra- and interrater reliabilities for the full scale (KVIQ- ${ }_{20}$ ), as well as the subscales (KVIQ-P ${ }_{10 \mathrm{~V}}$ and KVIQ-P ${ }_{10 \mathrm{~K}}$ ), in persons with stroke, as well as healthy control participants. Moreover, 
internal consistencies were good and matched the criteria for useful scales for research and clinical purposes in both groups. There were no statistically significant differences in the KVIQ-P performance between the groups.

Our results were similar to those reported for the original English version of the KVIQ- $\mathrm{P}_{20}$, KVIQ- $\mathrm{P}_{10 \mathrm{~V}}$ and KVIQ-P ${ }_{10 \mathrm{~K}}$ in Canadians with stroke ${ }^{1}$, both in terms of performance and psychometrics. Similar methodologies were used in the original Canadian study and in our protocol. Our study tested more subjects $(n=40)$ than the Canadian study $(n=19)$, although the latter included more controls (46 in theirs vs. 31 in ours). We found similar results in the control group when compared with the original study. The present study, therefore, not only expands the evidence base for the psychometric properties of the KVIQ to the Brazilian-Portuguese context, but further enhances the specific evidence base for KVIQ data in persons with stroke. As such, it adds an important component to the literature reporting reliability and consistency of the $\mathrm{KVIQ}_{20}$ or its subscales in participants with multiple sclerosis ${ }^{23}$, Parkinson's disease ${ }^{9}$ and a mixed sample of participants with stroke, brain tumors, multiple sclerosis or Parkinson's disease ${ }^{24}$. These previous studies in populations with different neurological conditions reported intra-rater reliability, not inter-rater reliability. The present research contributes to this body of research and, most critically, does so for a sizable and well-controlled stroke population. Overall, these results suggest that the $\mathrm{KVIQ}_{20}, \mathrm{KVIQ}_{10 \mathrm{~V}}$ and $\mathrm{KVIQ}_{10 \mathrm{~K}}$ are reliable and consistent across cultures, in people with diverse neurological conditions, as well as in healthy participants.

Mirroring the findings for intra-rater and inter-rater reliabilities, the internal consistencies of the Brazilian-Portuguese version of the $\mathrm{KVIQ}_{20}$ and of its subscales were very good (Cronbach- $\alpha$ ranged from 0.94 to 0.97 across the main scale, subscales and groups) and fulfilled the quality criterion for reliable comparisons between clinical groups as specified by Bland and Altman ${ }^{20}$. Again, this suggests that our version of the KVIQ-P has excellent psychometric characteristics overall.

Most of our participants in the stroke group were younger than 65 years, had more than eight years of education and normal MMSE scores. In other words, most participants were relatively young, educated, and did not have overt cognitive impairments, which means that, despite the stroke, they were a homogeneous and favorable group for motor imagery tasks. These results indicate that, in contrast to many other cerebral capacities, motor imagery may not suffer expressive damage in participants with a profile similar to those included in this study, or may recover due to plastic mechanisms in such individuals. Therefore, motor imagery interventions may be useful in stroke rehabilitation when other motor and sensorybased rehabilitation systems are compromised, at least in a subset of subjects.

The current research has limitations. While the sample size is not as large and diverse as one might consider ideal, the number of participants tested here was larger than in all other reports of the $\mathrm{KVIQ}_{20}$ performances in participants with stroke. Secondly, the first rater tested participants in person, while the second rater rated performances videotaped by the first rater. This approach has been used in other studies $^{25,26}$. Thirdly, specific lesion data was not available and therefore we were unable to comment on the influence of the lesion site on the KVIQ performance. A better understanding of the impact of different lesion sites on motor imagery performance is clearly an important question that should be addressed in the future.

Former work ${ }^{27}$ described and translated the $\mathrm{KVIQ}_{20}$ into Portuguese, but this version had not previously been validated. Likewise, other tools to quantify motor imagery like the Motor Imagery Questionnaire and the Vividness Motor Imagery Questionnaire were not yet validated in Portuguesespeaking individuals.

\section{CONCLUSIONS}

The present study shows excellent psychometric characteristics for the Brazilian-Portugese version of the KVIQ and, as such, strengthens the portfolio of tools available to study motor imagery in the Brazilian population. Moreover, the study expands a small body of research, conducted in various countries, suggesting that motor imagery in persons with stroke might be comparable to control populations ${ }^{28}$. This has implications for rehabilitation protocols. Specifically, it is well accepted that imagery reinforces the neural activity involved in the execution of motor programs, and can therefore enhance motor performance and learning ${ }^{29,30}$. Reliable tools to assess motor imagery are key to trials aiming at evidence-based imagery protocols to improve clinically meaningful outcomes in people with stroke. The excellent psychometric properties of the KVIQ- $\mathrm{P}_{20}$, KVIQ- $\mathrm{P}_{10 \mathrm{~V}}$ and KVIQ- $\mathrm{P}_{10 \mathrm{~K}}$ should be taken into consideration during the design of such trials.

\section{References}

2. Sterr A, Conforto AB. Plasticity of adult sensorimotor system in severe brain infarcts: challenges and opportunities. Neural Plast. 2012;2012:970136. https://doi.org/10.1155/2012/970136

3. Braun SM, Beurskens AJ, Borm PJ, SchackT, Wade DT. The effects of mental practice in stroke rehabilitation: a systematic review. Arch Phys Med Rehabil. 2006;87(6):842-52. https://doi.org/10.1016/j.apmr.2006.02.034 
4. Mulder T. Motor imagery and action observation: cognitive tools for rehabilitation. J Neural Transm (Vienna). 2007;114(10):1265-78. https://doi.org/10.1007/s00702-007-0763-z

5. Szameitat AJ1. Shen S, Sterr A. Motor imagery of complex everyday movements. An fMRI study. Neuroimage. 2007;15(34):702-13.

6. Hanakawa T, Dimyan MA, Hallett M. Motor planning, imagery, and execution in the distributed motor network: a time-course study with functional MRI. Cereb Cortex. 2008;18(12):2775-88. https://doi.org/10.1093/cercor/bhn036

7. Ueno T, Inoue M, Matsuoka T, Abe T, Maeda H, Morita K. Comparison between a real sequential finger and imagery movements: an FMRI study revisited. Brain Imaging Behav. 2010;4(1):80-5. https://doi.org/10.1007/s11682-009-9087-y

8. Malouin F, Richards CL, Durand A, Doyon J. Clinical assessment of motor imagery after stroke. Neurorehabil Neural Repair. 2008;22(4):330-40. https://doi.org/10.1177/1545968307313499

9. Randhawa B, Harris S, Boyd LA. The Kinesthetic and Visual Imagery Questionnaire is a reliable tool for individuals with Parkinson disease. J Neurol Phys Ther. 2010;34(3):161-7. https://doi.org/10.1097/NPT.0b013e3181e1aa71

10. Instituto Brasileiro de Geografia e Estatística - IBGE. Pesquisa nacional por amostras de domicílio contínua. Rio de Janeiro: Instituto Brasileiro de Geografia e Estatística; 2015 [cited jan 2015]. Available at: https://www.ibge.gov.br/home/estatistica/pesquisas/ pesquisa_resultados.php?id_pesquisa $=149$

11. Almeida OP. [The mini-mental state examination and the diagnosis of dementia in Brazil]. Arq Neuropsiquiatr. 1998;56(3B):605-12. Portuguese. https://doi.org/10.1590/S0004-282X1998000400014

12. Groot MH, van Campen JP, Moek MA, Tulner LR, Beijnen JH, Lamoth $\mathrm{CJ}$. The effects of fall-risk-increasing drugs on postural control: a literature review. Drugs Aging. 2013;30(11):901-20. https://doi.org/10.1007/s40266-013-0113-9

13. Dulin D, Hatwell Y, Pylyshyn Z, Chokron S. Effects of peripheral and central visual impairment on mental imagery capacity. Neurosci Biobehav Rev. 2008;32(8):1396-408. https://doi.org/10.1016/j.neubiorev.2008.04.007

14. American Psychiatry Association. Diagnostic and statistical manual of mental disorders. DSM - 5. Washington, DC: American Psychiatry Association; 2013

15. Shumway-Cook A, Baldwin M, Polissar NL, Gruber W. Predicting the probability for falls in community-dwelling older adults. Phys Ther. 1997;77(8):812-9. https://doi.org/10.1093/ptj/77.8.812

16. Sperber AD. Translation and validation of study instruments for cross-cultural research. Gastroenterology. 2004;126(1 Suppl 1):S124-8. https://doi.org/10.1053/j.gastro.2003.10.016

17. Orfale AG, Araújo PM, Ferraz MB, Natour J. Translation into Brazilian Portuguese, cultural adaptation and evaluation of the reliability of the disabilities of the arm, shoulder and hand questionnaire. Braz J Med Biol Res. 2005;38(2):293-302. https://doi.org/10.1590/S0100-879X2005000200018

18. Wild D, Grove A, Martin M, Eremenco S, McElroy S, Verjee-Lorenz A et al. Principles of good practice for the translation and cultural adaptation process for participants-reported outcomes (PRO) measures: report of the ISPOR Task Force for Translation and Cultural Adaptation. Value Health. 2005;8(2):94-104. https://doi.org/10.1111/j.1524-4733.2005.04054.x

19. Cha ES, Kim KH, Erlen JA. Translation of scales in cross-cultural research: issues and techniques. J Adv Nurs. 2007;58(4):386-95. https://doi.org/10.1111/j.1365-2648.2007.04242.x

20. Bland JM, Altman DG. Cronbach's alpha. BMJ. 1997;314(7080):572. https://doi.org/10.1136/bmj.314.7080.572

21. Fleiss JL. The Design and analysis of clinical experiments. J Clin Exp Neuropsychol. 2015;37:92-101.

22. Clark LA, Watson D. Constructing validity: basic issues in objective scale development. Psychol Assess. 1995;7(3):309-19. https://doi.org/10.1037/1040-3590.7.3.309.

23. Tabrizi YM, Zangiabadi N, Mazhari S, Zolala F. The reliability and validity study of the Kinesthetic and Visual Imagery Questionnaire in individuals with multiple sclerosis. Braz J Phys Ther. 2013;17(6):588-92. https://doi.org/10.1590/S1413-35552012005000124

24. Schuster C, Lussi A, Wirth B, Ettlin T. Two assessments to evaluate imagery ability: translation, test-retest reliability and concurrent validity of the German KVIQ and Imaprax. BMC Med Res Methodol. 2012;12(1):127. https://doi.org/10.1186/1471-2288-12-127

25. Jensen EA, Panitch H, Feng R, Moore PE, Schmidt B. Interobserver reliability of the respiratory physical examination in premature infants: a multicenter study. J Pediatr. 2016;S0022-3476:30641-2.

26. Yang Y, Wang Y, Zhou Y, Chen C, Xing D. Reliability of functional gait assessment in patients with Parkinson disease: interrater and intrarater reliability and internal consistency. Medicine (Baltimore). 2016;95(34):e4545. https://doi.org/10.1097/MD.0000000000004545

27. Silva, Larissa Rebola Volpi da et al. [Trainability for imagery capacity in individuals post stroke]. Rev Educ Fis UEM. 2012;23:389-98. Portuguese. https://doi.org/10.4025/reveducfis.v23i3.16747

28. Dickstein R, Deutsch JE. Motor imagery in physical therapist practice. Phys Ther. 2007;87(7):942-53. https://doi.org/10.2522/ptj.20060331

29. Braun S, Kleynen M, Schols J, Schack T, Beurskens A, Wade D. Using mental practice in stroke rehabilitation: a framework. Clin Rehabil. 2008;22(7):579-91. https://doi.org/10.1177/0269215508090066

30. Malouin F, Richards CL, Durand A, Doyon J. Added value of mental practice combined with a small amount of physical practice on the relearning of rising and sitting post-stroke: a pilot study. J Neurol Phys Ther. 2009;33(4):195-202. https://doi.org/10.1097/NPT.0b013e3181c2112b 